\title{
Research on Gait-Based Gender Classification via Fusion of Multiple Views
}

\author{
Zhang De \\ School of Electrical and Information Engineering, Beijing University of Civil \\ Engineering and Architecture, Beijing, 100044, P.R. China \\ zhangde@bucea.edu.cn
}

\begin{abstract}
Automatic gender classification of an individual can be very useful in video-based surveillance systems and human-computer interaction systems. Currently, gait from a single viewpoint has been used to recognize the gender of a person. Considering the multiple cameras used in real environments, we investigate gender classification from human gait by using multi-view fusion, a relatively understudied problem. In this paper, we present a new approach to integrate information from multi-view gait at the feature level. First, gait energy images (GEI) are constructed from the video streams for different viewpoints. Then, the feature fusion is performed by putting GEI images and camera views together to generate a third-order tensor (x, y, view). A multi-linear principal component analysis (MPCA) is employed to reduce dimensionality of the tensor objects which integrate all views. The proposed fusion scheme is tested on CASIA database and compared with other fusion methods. The experimental results show that MPCA based feature fusion is quite effective for multi-view gait based gender classification.
\end{abstract}

Keywords: Gait, Gender recognition, Multi-view fusion

\section{Introduction}

Gait is the manner of walking as defined in Webster's New Collegiate Dictionary. However, human gait is more than that: it is an identifying feature of a person that is determined by the weight, limbs length, and habitual posture. Hence, it is reasonable to use gait as a biometric measure to recognize known people or determine the gender of an individual. Human walking patterns contain informative and stylistic variations. Even from moving light displays (MLD), people can recognize the gender of walkers [1-4] or identify a person [5-6].

Gender classification is an important visual task for human beings since a typical social category is gender. Automatic gender classification is helpful in many applications, such as security and surveillance in public areas and customer analys is for commercial purposes. Human faces are commonly used for the determination of a person's gender. This problem has been studied on large datasets recently [12-14]. However, in many real-world video surveillance systems, it is hard to capture face information at a high enough resolution if the person is far away from the camera. This will lead to an unreliable identification of the gender of a person. In these situations, human gait can provide an alternative cue because it is easy to be detected and measured at a distance. This biometric modality also has the advantages of being non-contact and non-invasive.

There exists several computer vision systems developed for gait based gender recognition [16-19]. However, these systems depend on only the side view and are susceptible to viewing angle variation. Therefore, to develop a robust and accurate gender classification system based on gait has become an important challenge and research direction. This paper proposes a new approach which integrates multi-view 
gait sequences to carry out gender classification. This approach can be used in practice conveniently because in many surveillance environments there are multiple cameras fixed at different viewpoints, which makes it possible to acquire gait sequences from multi-view directions.

In this work, features are obtained using gait energy image (GEI) from each view separately. For the fusion of multiple views at the feature level, we augment the dimension of sample space by taking camera views into account. Given the GEI of size $m$-by- $n$ and the number of different camera views $c$, the original feature point in the sample space becomes a tensor object of size $m$-by- $n$-by-c. Then, in this augmented sample space, a feature extraction method called multi-linear principal component analysis (MPCA) [7] is implemented. The proposed fusion scheme is tested on CASIA Gait Dataset B [26] which contains eleven different camera views.

The rest of the paper is organized as follows. Section 2 introduces related work on gait based gender classification and multi-view gait fusion. Section 3 describes the details of the proposed approach and some other fusion methods to be compared. In Section 4, testing experiments are presented and the experimental results are compared and discussed. Section 5 concludes the paper.

\section{Related Work}

The study of recognizing gender from human gait begins with the attention on moving light displays (MLD), since point-light displays provide an ideal way to study the contribution of motion to the perception of biological movements [9]. An experiment on a small database which contains 6 walkers ( 3 females and 3 males) of approximately the same height and weight is described in [3]. It is demonstrated that human observers can discriminate the gender of walkers shown in side view with a performance of $63 \%$ correct recognition. Temporal and spatial factors are explored in a further study [1].

Pattern recognition approaches were developed for gait based gender classification in the late 1990s. In [10], a two-stage PCA framework is proposed to transform human walking data into a representation that allows such data to be applied to simple linear classification methods from statistics and pattern recognition. The dataset used in [10] consisted of three-dimensional motion-capture trajectories of 40 walkers (20 females and 20 males). In [15], a three-mode expressive-feature model for recognizing gender from point-light displays is presented and tested on the same dataset mentioned above. This method factored prototype female and male walkers into their three-mode principal components and learned the expressive feature weights from training data automatically. A 95.5\% accuracy achieved in [15] shows that automatic gait-based gender classification is feasible.

However, the aforementioned studies can hardly be applied in surveillance systems because it is unreasonable to attach retro-reflective markers to the body of a walker in real applications. So, video based approaches have been developed in recent years when the computer speed on processing image sequences became high enough. But, most researchers focus on human identification from gait video sequences. The problem of gender classification from human gait remains relatively unstudied although there are some studies appearing in [16-19].

In [16], a new gait appearance representation is proposed by proportionally dividing the silhouette into seven regions. For each of them, an ellipse is fitted and several parameters related to the ellipses are taken as features. These features are applied to gender classification and experiments are done on a small gait database (10 females and 14 males). A recognition rate of $84.5 \%$ is achieved under randomperson test condition. Yoo et al. use a sequential set of $2 \mathrm{D}$ stick figures to represent 
the gait signature [17]. Both SVM and neural networks are employed to carry out gender classification. The results show that SVM outperforms neural networks for the given task. The best recognition rate from a gait database including 84 males and 16 females reaches $96 \%$ under random-sequence test condition. The randomsequence test means the sequences from the same person, though not the same sequences, are in both the training and the testing data. In [18], Li et al. separate the averaged gait silhouette image into seven components and study the effectiveness of these components for human gender recognition. Their experiments show that two components, the trunk and the front-leg are usually important for gender recognition. In [19], Yu et al. conduct a more reasonable segmentation on human gait silhouette and assign different weights to different components according to a priori psychological experiment when classifying one's gender.

The aforementioned experiments on recognizing gender are implemented using a side view presentation of the walkers to observers. In recent years, multi-view gait recognition has been studied and the fusion of multi-view gait sequences generates improved results $[11,21]$. In this work, we develop such fusion methods for gender classification. Wang et al. [11] present a fusion scheme of multi-view gait sequences. They used CASIA gait database (Dataset B) acquired at eleven different views. Dempster-Shafer rule used at the decision level produced a great improvement in comparison with single view based gait recognition. In [21] Huang et al. calculate the weights of each individual view by minimizing the probability of inaccurate classification and use these different weights to sum the distances between the test subject and the reference subject for each view. They used CMU MoBo database and chose five out of six available viewing directions.

A multi-view fusion system for gait based gender classification is first presented in [22]. The front-view, back-view and side-view were combined and the feature extraction method proposed in [16] was applied to these viewpoints. The fusion was implemented at the score level using sum rule and it achieved a better performance than any of the three single views. Actually, the CASIA gait database (Dataset B) used in [22] includes eleven different camera views. But the gait representation proposed in [16] is not appropriate to be applied to other inclined viewing angles because the human body doesn't look symmetric for these angles.

\section{Technical Approach}

We use the fusion scheme at the feature level proposed in our previous work [26] to carry out gender classification with multi-view gait. Gait energy image (GEI) is first constructed from video sequences as the gait template for each camera view. Assume that the size of GEI is $m$-by- $n$ and the number of available camera views is $c$. Then a tensor object, $(x, y$, view $)$, of size $m$-by- $n$-by- $c$ can be rendered by putting GEI images from all the views together. Multi-linear principal component analysis (MPCA) is employed to extract synthetic features from this tensor space and subsequently, the feature fusion is implemented. Figure 1 illustrates this fusion system.

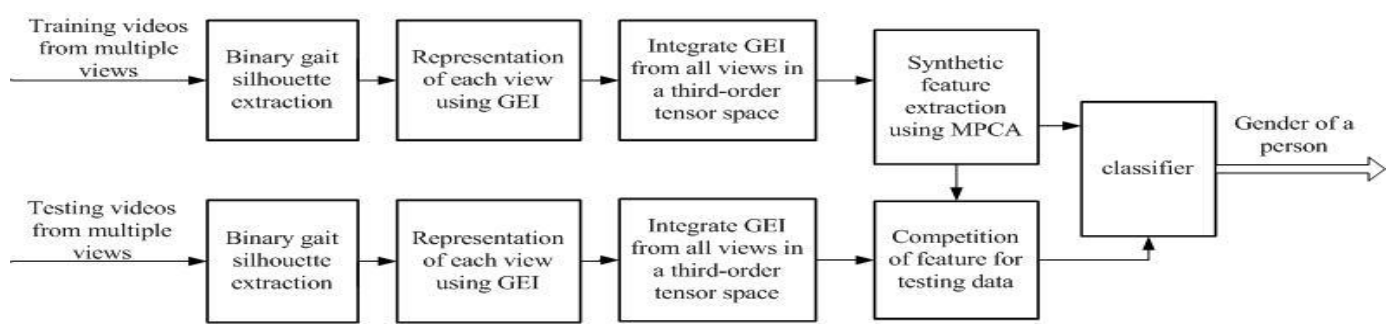

Figure 1. The Proposed Feature Level Fusion System 


\subsection{GEI Construction}

As summarized in [20], there are many approaches to extract gait features from the side view $\left(90^{\circ}\right)$ by using the silhouette or designing a model. We need a representation that can be generalized to other view angles while characterizing gait effectively. Gait energy image (GEI) is an effective representation of gait proposed by Han and Bhanu [23]. It reflects both static stance information of silhouettes and dynamic shape changing information over a gait cycle. Yu et al. [8] use it for eleven different views in CASIA gait database (Dataset B) to evaluate the effect of viewing angle variation on gait recognition. Moreover, GEI of side view is used to classify gender in [18-19].

In this work we also take GEI as the representation of gait to test the performance of recognizing the gender of an individual by fusing multi-view gait. In order to construct GEI, several steps are required: (a) Silhouette extraction. The binary silhouettes are extracted from original walking videos by means of the technique of background subtraction. We use the mean value of multiple frames to update the background. (b) Normalization. It includes scaling the foreground regions to the same height while keeping the ratio of their height to width and moving them to the center of silhouette images. (c) Detection of gait period. We use the approach proposed in [24] to estimate gait periodicity, $N_{\text {gait }}$. We count the number of foreground pixels in the bottom half of the silhouette in each frame overtime, $N_{f}(t)$. This number reaches the maximum when the two legs are farthest apart and drop to a minimum when the legs overlap. But a problem with this strategy is that there exists some noise in the signal $N_{f}(t)$, as shown in Figure 2(a). Hence, we use the autocorrelation technique to remove noise and estimate the length of a gait cycle accurately.

We identify the gait cycle length $N_{\text {gait }}$ by calculating the autocorrelation $R_{w}[\mathrm{~m}]$ of the signal $w(t)$ which is derived from $N_{f(t)}$ by subtracting its mean value and dividing by its range. It is expressed as:

$$
w(t)=\frac{N_{f}(t)-\operatorname{mean}\left(N_{f}(t)\right)}{\max \left(N_{f}(t)\right)-\min \left(N_{f}(t)\right)}
$$

The autocorrelation of $w(t)$ is defined as:

$$
R_{w}[m]=E\{w(t) w(t+m)\}
$$

Where $E\{\cdot\}$ denotes the expectation operator. The cycle length $N_{\text {gait }}$ is calculated as the smallest $m$, other than $m=0$, at which there is a local maximum of $R_{w}[m]$. As shown in Figure 2(b), the determination of $N_{\text {gait }}$ from the autocorrelation function is unambiguous despite of the noisy original sequence.

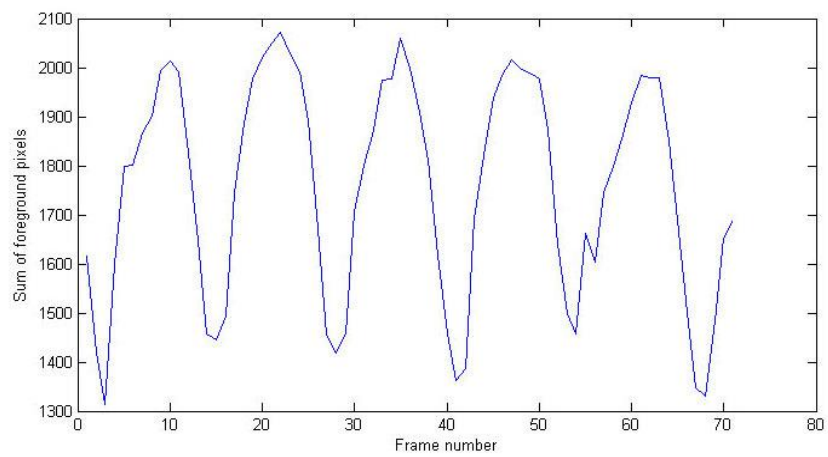

(a) 


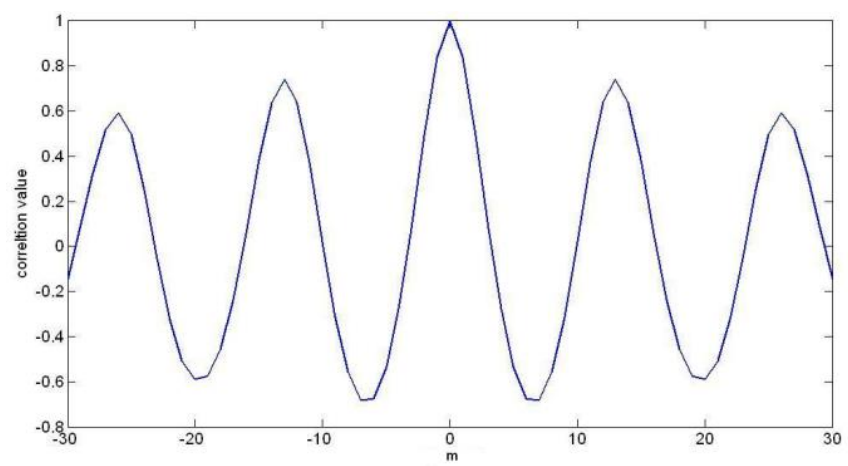

(b)

This method of detecting gait period works well for the gait sequences from the view of $90^{\circ}$ according to our experiments, since the movement of the two legs can be captured quite clearly for this view. An example of gait sequences from different views is shown with normalized silhouette images in Figure 3. Because the cameras record human walking simultaneously in our database, the values of $N_{\text {gait }}$ for different camera views are the same for one walk. The frame number labels obtained from the original videos are used to synchronize the starting frame of a gait cycle for all the views in one walk. We extract one cycle of frames from the synchronized silhouettes for each camera view and the grey-level gait energy image (GEI) for each view can be calculated as:

$$
G(x, y)=\frac{1}{N_{\text {gait }}} \sum_{t=1}^{N_{\text {gait }}} B_{t}(x, y)
$$

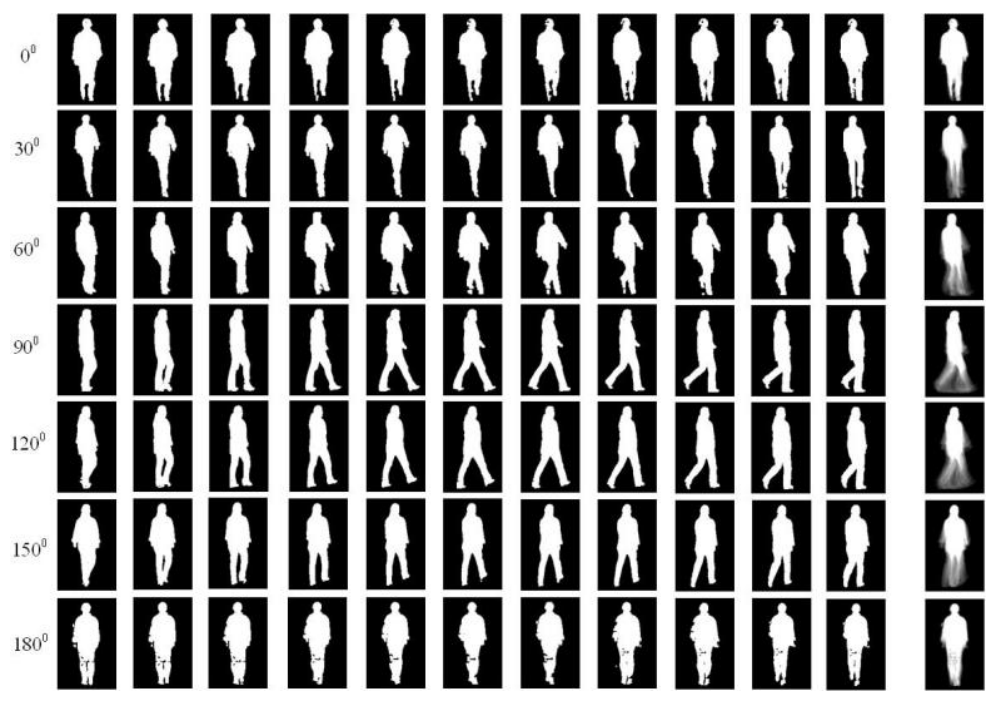

Figure 3. Examples of Normalized and Centered Silhouette Frames from different Views for One Walk. The Leftmost Column Lists the Degrees of Viewing Angles. The Rightmost Image in each Row is the Corresponding Gait Energy Image (GEI)

Where $B_{t}(x, y)$ is the silhouette image at time $t$ in a gait period. Figure 3 shows some silhouette samples within a gait cycle from different views in our database and the right-most images are the corresponding GEIs. As can be seen, GEI contains spatio-temporal information of a gait period in a compact way, no matter what the view angle is. 


\subsection{Fusing Multi-View Gait}

The fusion scheme used in this paper is at the feature level. It treats all the GEIs from different camera views as a data sample, which is a third-order tensor. The spatial row space and column space of GEI as well as camera view space account for the three modes.

GEIs are derived from a gait cycle as described above. We construct GEIs for all the different views as illustrated in Figure 3. Considering a walk recorded by cameras from different view angles simultaneously, we pick one gait cycle in this walk and obtain the corresponding GEIs for all views. A multi-view fusion is realized at the feature level when concatenating these two-dimensional GEIs in a three-dimensional space. Hence, we get the new integrated data samples in a thirdorder tensor space as shown in Figure 4.

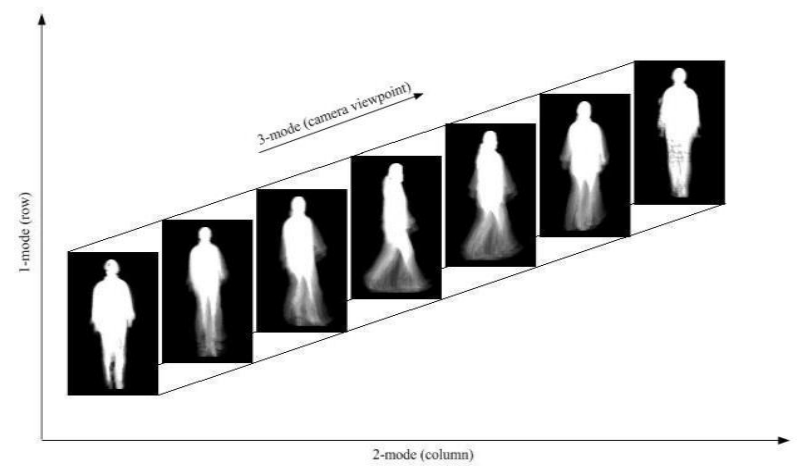

Figure 4. Illustration of GEls from Multiple Views as a Third-Order Tensor

\subsection{Feature Learning Using MPCA}

Multi-linear principal component analysis (MPCA) is a direct extension of principal component analysis (PCA) to the multi-linear case. A MPCA framework is proposed in [7] for feature selection from tensor objects. The input data samples are centered as in PCA, the projection is orthonormal and the projected feature is a tensor of the same order as the data samples with reduced dimension.

The basic idea of multi-linear PCA formulation follows the classical PCA paradigm. Given $M$ training tensor samples $\left\{\chi_{1}, \chi_{2}, \ldots, \chi_{M}\right\}$ from an $N$-order dataset, compute its mode- $N$ mean $\bar{\chi}^{(N)}$. Mode denotes a dimension in the data space. The last mode is defined to indicate how many samples are there in a dataset. The training data is centered by subtracting $\bar{\chi}^{(N)}$ from each tensor sample. Let $\chi_{c}$ denote the centered training samples. It can be decomposed using higher-order singular value decomposition (HOSVD) as:

$$
\chi_{c}=S \times{ }_{1} U^{(1)} \times{ }_{2} U^{(2)} \cdots \times{ }_{2} U^{(N)}
$$

Where $S \in R^{I_{1} \times I_{2} \times \cdots \times I_{N}}$ is the core tensor of which the subtensors $S_{i_{n}=\alpha}$ have the property of all-orthogonality and ordering based on the Frobenius-norms $\left\|S_{i_{n}=\alpha}\right\|$, and $U^{(N)}$ is an orthogonal $I_{n} \times I_{n}$ matrix.

As in singular value decomposition (SVD) truncation for PCA, the HOSVD of $\chi_{\text {c }}$ is truncated by keeping in the first $R_{n}$ columns for the basis matrix $U^{(n)}$ in each mode $n$ to produce $\tilde{U}^{(n)}$. The tensor projection using the collection of these $\prod_{n=1}^{N-1} R_{n}$ basis matrix is expressed as: 


$$
\tilde{p}_{-N}=\times_{1} \tilde{U}^{(1) T} \times{ }_{2} \tilde{U}^{(2) T} \cdots \times_{N-1} \tilde{U}^{(N-1) T}
$$

Where $T$ is the matrix transpose operator. Some examples of basis matrix generated CASIA gait database (Dataset B) are shown in Figure 11.

MPCA is an effective approach to deal with tensor data. In this paper, we apply it to reduce dimensionality of the tensor samples constructed from multiple views. The output of MPCA is the feature set that is fused from multi-view gait.

\subsection{Related Fusion Schemes}

In this paper, we also compare the proposed feature level fusion scheme with the related fusion schemes at the decision level described in [25, 27].

Three fusion strategies are used in [25]. The first is the voting rule which gives the final decision according to the majority of votes. The second is the weighted voting rule which is actually a variant of the voting rule. Each classifier is assigned an individual weight before voting. The last is the Bayes combination rule. Bayes formula shows how to calculate the posteriori probability of an observation when the prior probabilities and the conditional densities are known. In our case, $P_{m}\left(v_{i j}\right)$ denotes the probability that the $i$-th person is classified as male from the $j$-th classifier. It can be defined as:

$$
P_{m}\left(v_{i j}\right)=\frac{N_{m}}{N_{t}}
$$

Where $N_{m}$ is the number of votes on male and $N_{t}$ is the number of total votes. Likewise, $P_{f}\left(v_{i j}\right)$ denotes the probability being classified as female. Then, the fusion method can result in the probability $p_{m_{i}}$ of recognizing a walker as a male with an expression based on Bayes formula:

$$
p_{m_{i}}=\frac{P(m) \sum_{j} P_{m}\left(v_{i j}\right)}{P(m) \sum_{j} P_{m}\left(v_{i j}\right)+P(f) \sum_{j} P_{f}\left(v_{i j}\right)}
$$

Where $P(m)$ is the percent that male subjects take in the database and $P(f)$ is the percent of females. They are the prior probabilities of the two classes. $\sum_{j}$ denotes summing up the results from all the classifiers.

\section{Experiments}

\subsection{Database}

The CASIA gait database (Dataset B) [26] is a large multi-view database for gait analysis which includes 93 males and 31 females. All of the 31 females are from East Asia. This database is used for the study of gender classification in $[19,22]$. There are eleven different camera views in this database. For each viewpoint, there are six video sequences per person. We selected 31 males randomly to match the total number of females in our experiments as in the case in [19]. All of the subjects we selected from this database are East Asians.

\subsection{Experimental Results}

We performed the MPCA-based feature fusion scheme and compared the results with single-view based gender classification results and decision-level fusion (mentioned in Section 3.4) results respectively. 
We use all the 31 females and randomly select 31 males from CASIA Gait Dataset B for this experiment as [19]. There are eleven different camera views in the database. All of these subjects we select are East Asian. Each of them has six walks used in the experiments. For each walk, we have eleven video sequences with different view angles. We select a gait cycle and construct a GEI from every video sequence. A tensor object is generated from a given walk by combining these eleven GEIs as mentioned before. Totally, we have $31 \times 2 \times 6=372$ tensor samples and the size of each sample is $155 \times 100 \times 11$. We divide these samples into 31 groups. Each group consists of the entire data from a male subject and a female subject. We take one group in turn as testing data and the remaining as training data. So, it iterates 31 times and we report the average correct classification rate (CCR) of these 31 groups as the final result.

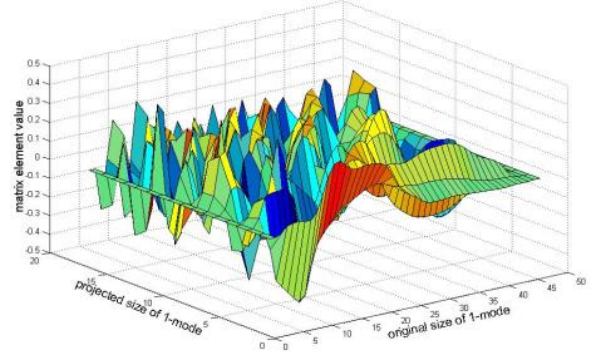

(a)

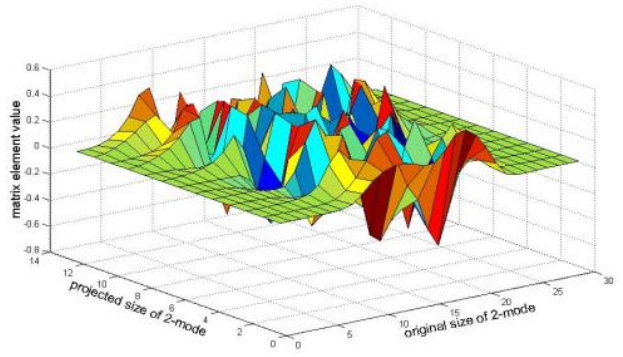

(b)

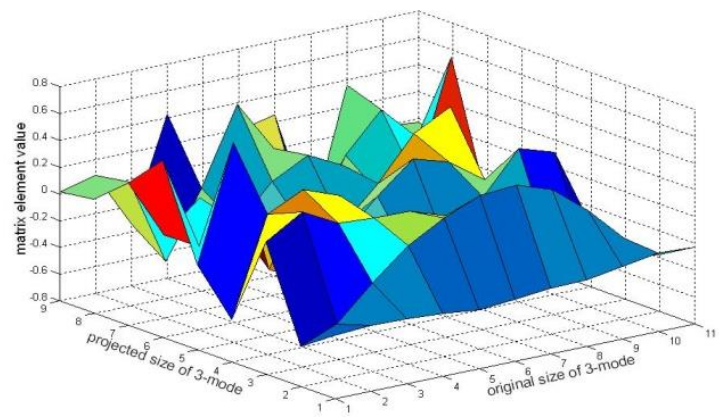

(c)

Figure 5. (a) 1-Mode Projection Matrix, (b) 2-Mode Projection Matrix, (c) 3Mode Projection Matrix

During each time of iteration, there are $30 \times 2 \times 6=360$ tensor samples in training data and MPCA is applied to extract features. In order to avoid the error of out of memory, we down sample GEI images to reduce the size of tensor samples to $47 \times 30 \times 11$. Take one iteration as an example to show the parameters here. The number of eigenvectors kept in each mode is: $\mathrm{P}_{1}=17, \mathrm{P}_{2}=13$, and $\mathrm{P}_{3}=9$, determined by setting $Q^{(1)}=Q^{(2)}=Q^{(3)}=Q=0.97$, which captures approximately $92 \%$ of the total variations of training data in the projected tensor subspace. For other iterations, the value of $Q$ doesn't change but the number of eigenvectors kept has slight differences because the training data is different for each iteration. In Figure 5, we illustrate the multi-linear projection matrices in one iteration. 


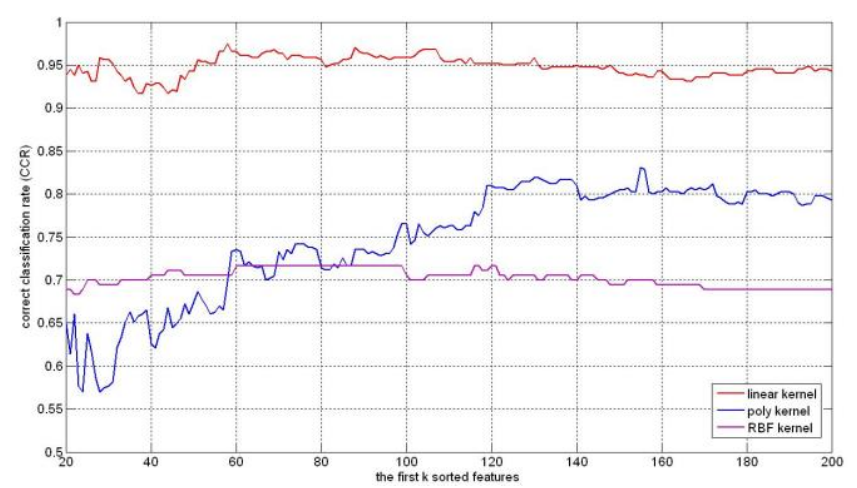

Figure 6. CCR Versus MPCA Dimensionality Reduction for Different SVM Kernels

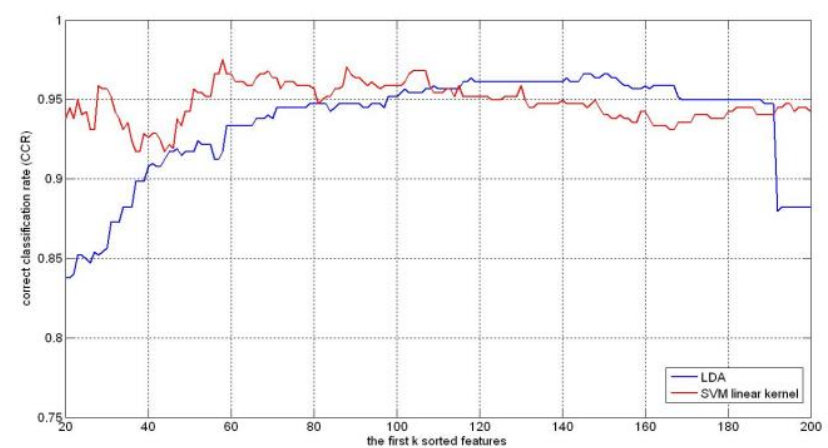

\section{Figure 7. CCR Versus MPCA Dimensionality Reduction for Different Methods}

For each iteration, the projected feature is a third-order tensor. Take the first iteration as an example. The size of the tensor is $17 \times 13 \times 9$. We rearrange it into a feature vector of size 1,989 which is sorted in a descending order of class discriminability. As this problem of classification is supervised, the class discriminating power is defined to be the ratio of the between-class scatter over the within-class scatter. We consider support vector machines (SVM) as the classifier. The classification performance varies with the dimensionality of the MPCA feature vector as shown in Figure 6. Figure 6 also illustrates the CCRs for different SVM kernels. We use the $2_{\text {nd }}$ degree polynomial kernel and set the radius width with 4 for RBF kernel. These values are the best with around zero training loss. The size of feature vector, $k$, starts from 20 because the results do not converge when $k$ is less than 20 in our experiments.

It is observed that the linear kernel performs much better than polynomial kernel and RBF kernel. This shows that the distinction between genders is consistent with a linear boundary. So we carry out further experiments with linear discriminant analysis (LDA) to confirm this inference. Euclidean distance based nearest-neighbor classifier is used to determine the gender of a testing subject. In Figure 7 we compare the results from LDA and SVM with linear kernel. It shows that these two methods can reach a similar performance for gender classification. SVM with linear kernel achieves the best CCR at a smaller $k$ than LDA. Also, it performs slightly better than LDA as illustrated in Figure 7. Therefore, we choose SVM with linear kernel as the classifier in other experiments.

From Figure 6 and Figure 7, it can be observed that the best CCR for SVM is $98.1 \%$, which indicates that the proposed fusion scheme at the feature level works well. The results from the single views are listed in Table 1 and compared with the best CCR from the proposed fusion scheme. PCA is used to reduce the dimension of 
GEI for any individual view. We select $90 \%$ of the total cumulative energy of eigenvectors empirically. The classification results are obtained by SVM with linear kernel. The improved result by this proposed fusion scheme is shown in the bottom row of Table 1. It is much better than the results from single views. From Table 1, it is also shown that the CCR at the viewing angle of $90^{\circ}$ is the best, indicating the side view is the most discriminative for GEI based gender classification.

\section{Table 1. Result of Gender Recognition Using SVM with Linear Kernel}

\begin{tabular}{|c|c|}
\hline Viewing angle & CCR \\
\hline \hline $0^{0}$ & $72.3 \%$ \\
\hline $18^{0}$ & $73.4 \%$ \\
\hline $36^{0}$ & $76.4 \%$ \\
\hline $54^{0}$ & $91.7 \%$ \\
\hline $72^{0}$ & $93.0 \%$ \\
\hline $90^{0}$ & $94.8 \%$ \\
\hline $108^{0}$ & $92.1 \%$ \\
\hline $126^{0}$ & $84.3 \%$ \\
\hline $144^{0}$ & $78.1 \%$ \\
\hline $162^{0}$ & $75.6 \%$ \\
\hline $180^{0}$ & $74.2 \%$ \\
\hline Fusion of all views & $\mathbf{9 8 . 1 \%}$ \\
\hline
\end{tabular}

Next, we performed the fusion strategies mentioned in Section 3.4 on the same dataset (31 females and 31 males). For the decision level fusion, every single view is taken as a classifier. We firstly obtain the classification results from each view as done above. Then, for the voting rule, every single view corresponds to one vote in one given walk. For the weighted voting rule, the CCRs of single views listed in Table 1 are used as the weights. For Bayes combination rule, the $P(m)$ and $P(f)$ have the same value of 0.5 , since the numbers of male and female subjects are equal. In Table 2 we list the experimental results obtained with different fusion schemes. The proposed MPCA based feature fusion gives the best performance.

Table 2. Correct Classification Rate for different Fusion Schemes

\begin{tabular}{|c|c|c|c|}
\hline \multirow{2}{*}{ Voting rule } & $\begin{array}{c}\text { Weighted } \\
\text { voting rule }\end{array}$ & $\begin{array}{c}\text { Bayes } \\
\text { combination } \\
\text { rule }\end{array}$ & $\begin{array}{c}\text { FPCA-based feature } \\
\text { fusion }\end{array}$ \\
\hline \hline $96.5 \%$ & $96.5 \%$ & $96.8 \%$ & $\mathbf{9 8 . 1 \%}$ \\
\hline
\end{tabular}

\subsection{Comparison with Other Related Work}

In [22], Huang and Wang use the gait representation proposed in [16] and design a fusion of three views at the score level for gender classification. The dataset used in [22] includes 30 males and 30 females selected from CASIA gait database (Dataset B). In [19], Yu et al. divide GEI into five parts and assign different weights for each part. The experiments are carried out on a dataset of 31 males and 31 females selected from CASIA gait database (Dataset B). They also implement the method proposed in [18] on the same dataset. Both of them use only the side view. 
The existing gait based gender classification techniques are listed in Table 3 for comparison. It can be seen that our proposed fusion method outperforms the others.

Table 3. Results of Different Methods

\begin{tabular}{|c|c|c|c|}
\hline \multirow[b]{2}{*}{ Method } & \multicolumn{2}{|c|}{ Database } & \multirow[b]{2}{*}{ CCR } \\
\hline & Number of subjects & View angles & \\
\hline Proposed in [16] & $\begin{array}{c}14 \text { males and } 10 \\
\text { females }\end{array}$ & $90^{\circ}$ & $84.5 \%$ \\
\hline Proposed in [22] & $\begin{array}{c}30 \text { males and } 30 \\
\text { females }\end{array}$ & $0^{0}, 90^{0}, 180^{0}$ & $89.5 \%$ \\
\hline Proposed in [18] & $\begin{array}{c}31 \text { males and } 31 \\
\text { females }\end{array}$ & $90^{\circ}$ & $93.2 \%$ \\
\hline Proposed in [19] & $\begin{array}{c}31 \text { males and } 31 \\
\text { females }\end{array}$ & $90^{\circ}$ & $95.9 \%$ \\
\hline Proposed in this paper & $\begin{array}{l}31 \text { males and } 31 \\
\text { females }\end{array}$ & $\begin{array}{c}0^{0}, 18^{0}, 36^{0}, \ldots, \\
90^{0}, \ldots, 162^{0}, 180^{0}\end{array}$ & $98.1 \%$ \\
\hline
\end{tabular}

\subsection{Discussion}

The MPCA based multi-view fusion scheme concatenates GEIs of different views in a third-order tensor space. A requirement is that the frames from different camera views should be synchronized with time before calculating GEI. In this multi-view fusion, we integrate the stances captured by cameras from different view angles at the same time. The GEI is actually the averaged stance of a gait cycle. Therefore, the synchronization ensures the reasonableness and correctness of our proposed fusion scheme at the feature level. The experimental results show that the proposed feature fusion performs better than any single view and decision fusion.

\section{Conclusion}

In this paper, we introduce a multi-view fusion at the feature level for gait based gender classification. In this fusion scheme, the camera views are handled in a new way. By adding camera viewpoint to GEI image space, we generate third-order tensor samples in which different view angles are integrated. MPCA is then used to extract features from tensor objects. The experimental results show the improved performance of the proposed fusion scheme compared with single views and decision fusion schemes.

The fusion of multi-view gait is feasible and promising in real world applications. In public security environment, more and more cameras are put into surveillance systems. In such environment, many gait videos can be recorded from different camera views for gender classification or individual recognition. Gender is one of the important soft biometric traits. It can provide necessary information such as identifying a criminal suspect or counting customers of different genders for preferences of a store or a new product. Hence, the study of gender classification based on fusion of multi-view gait is definitely valuable for real world applications. It can be utilized as long as there is a surveillance system in a specific area.

\section{Acknowledgements}

This work was supported by Beijing Natural Science Foundation (4144070 and 4144072). 


\section{References}

[1] C. Barclay, J. Cutting and L. Kozlowski, "Temporal and spatial actors in gait perception that influence gender recognition", Perception and Psychophysics, vol. 23, no. 2, (1978), pp. 145-152.

[2] J. Cutting, D. Proffitt and L. Kozlowski, "A biomechanical invariant for gait perception", Journal of Experimental Psychology, vol. 4, no. 3, (1978), pp. 357-372.

[3] L. Kozlowski and J. Cutting, "Recognizing the sex of a walker from dynamic point-light display", Perception and Psychophisics, vol. 21, no. 6, (1977), pp. 575-580.

[4] G. Mather and L. Murdoch, "Gender discrimination in biological motion displays based on dynamic cues", Proceedings of the Royal Society of London, Series B, Biological Sciences, vol. 258, (1994), pp. 273-279.

[5] J. Cutting and L. Kozlowski, "Recognizing friends from their walk: Gait perception without familiarity cues", Bulletin of the Psychonomic Society, vol. 9, no. 5, (1977), pp. 353-356.

[6] T. Beardsworth and T. Buckner, "The ability to recognize oneself from a video recording of one's movements without seeing one's body", Bulletin of the Psychonomic Society, vol. 18, no. 1, (1981), pp. 19-22.

[7] H. Lu, K. N. Plataniotis and A. N. Venetsanopoulos, "MPCA: multilinear principal component analysis of tensor objects", IEEE Transactions on Neural Networks, vol. 19, no. 1, (2008), 18-39.

[8] S. Yu, D. Tan and T. Tan, "A framework for evaluating the effect of view angle, clothing and carrying condition on gait recognition", In Proceedings of IEEE International Conference on Pattern Recognition, (2006), pp. 441-444.

[9] G. Johansson, "Visual perception of biological motion and a model for its analysis", Perception and Psychophysics, vol. 14, no. 2, (1973), pp. 201-211.

[10] N. F. Troje, "Decomposing biological motion: A framework for analysis and synthesis of human gait patterns", Journal of Vision, vol. 2, no.5, (2002), pp. 371-387.

[11] Y. Wang, S. Yu, Y. H. Wang and T. Tan, "Gait recognition based on fusion of multi-view gait sequences", Advances in Biometrics, vol. 3832, (2005), pp. 605-611.

[12] B. Moghaddam and M. Yang, "Learning gender with support faces", IEEE Transactions on Pattern Analysis and Machine Intelligence,vol. 24, no. 5, (2002), pp. 707-711.

[13] Z. Yang and H. Ai, "Demographic classification with local binary patterns", Advances in Biometrics, vol. 4642, (2007), pp. 464-473

[14] G. Shakhnarovich, P. A. Viola and B. Moghaddam, "A unified learning framework for real time face detection and classification", In Proceedings of IEEE International Conference on Automatic Face and Gesture Recognition, (2002), pp. 14-21.

[15] J. W. Davis and H. Gao, "An expressive three-mode principal components model for gender recognition”, Journal of Vision, vol. 4, (2004), pp. 362-377.

[16] L. Lee and W. Grimson, "Gait analysis for Recognition and Classification", In Proceedings of IEEE International Conference on Automatic Face and Gesture Recognition, (2002), pp. 148-155.

[17] J. Yoo, D. Hwang and M. S. Nixon, "Gender classification in human gait with SVM", Advanced Concepts for Intelligent Vision Systems, vol. 3708, (2005), pp. 138-145.

[18] X. Li, S. J. Maybank, S. Yan, D. Tao and D. Xu, "Gait components and their application to gender recognition”, IEEE Transactions on Systems, Man and Cybernetics, Part C, vol. 38, no. 2, (2008), pp. 145-155.

[19] S. Yu, T. Tan, K. Huang, K. Jia and X. Wu, "A study on gait-based gender classification", IEEE Transactions on Image Processing, vol. 18, no. 8, (2009), pp. 1905-1910.

[20] M. S. Nixon and J. D. Carter, "Automatic recognition by gait", Proceedings of the IEEE, vol. 94, no. 11, (2006), pp. 2013-2024.

[21] X. Huang and N. V. Boulgouris, "Gait recognition using multiple views", In Proceedings of IEEE International Conference on Acoustics, Speech and Signal Processing, (2008), pp. 1705-1708.

[22] G. Huang and Y. Wang, "Gender classification based on fusion of multi-view gait sequences", In Proceedings of Aisan Conference Computer Vision, vol. 4843, (2007), pp. 462-471.

[23] J. Han and B. Bhanu, "Individual recognition using gait energy image", IEEE Transactions on Pattern Analysis and Machine Intelligence, vol. 28, no. 2, (2006), pp. 316-322.

[24] S. Sarkar, P. J. Phillips, Z. Liu, I. R. Vega, P. Grother and K. W. Bowyer, "The HumanID gait challenge problem: Data sets, performance, and analysis", IEEE Transactions on Pattern Analysis and Machine Intelligence, vol. 27, no. 2, (2005), pp. 162-177.

[25] D. Zhang and Y. Wang, "Gender recognition based on fusion of face and multi-view gait", Advances in Biometrics, vol. 5558, (2009), pp. 1010-1018.

[26] "Center for Biometrics and Security Research", CASIA. Http://www.cbsr.ia.ac.cn

[27] X. Jiang and S. Deng, "Optimal strategy for selling on group-buying website", Journal of Industrial Engineering and Management, vol. 7, no. 4, (2014), pp. 433-445. 